\title{
EL EFECTO DE LA SOBRECARGA CON CREATINA EN EL RENDIMIENTO FÍSICO EN SPRINT DE ATLETISMO, POSTERIOR A UN PROTOCOLO DE EJERCICIO INTERMITENTE Y PROLONGADO
}

\author{
Oscar Pablo Gutiérrez Sancho, José Moncada Jiménez y Walter Salazar Rojas \\ Escuela de Educación Fisica y Deportes, \\ Universidad de Costa Rica, San Jose, Costa Rica \\ E-mail:opgutier@hotmail.com
}

\begin{abstract}
Resumen
Gutiérrez Sancho, O. P., Moncada Jiménez, J., y Salazar Rojas, W. (2006). El efecto de la sobrecarga con creatina en el rendimiento físico en sprint de atletismo, posterior a un protocolo de ejercicio intermitente y prolongado. Revista de Ciencias del Ejercicio y la Salud, 4(1), 34-41. El estudio examina el efecto de la sobrecarga con Creatina (Cr) sobre el rendimiento en 6 sprints $(200 \mathrm{~m})$ de atletismo realizados a máxima intensidad, después de haber ocasionado la fatiga $(+1 \mathrm{hr})$ con ejercicio de carrera submáximo $(60-85 \%$ de $\left.\mathrm{FC}_{\text {máx }}\right)$. En un diseño de medidas repetidas y contrabalanceado 6 hombres activos pasaron por tres condiciones experimentales: condición control (CT); condición con creatina (CCr); y condición placebo (PL). En la condición CT no recibieron ningún tratamiento. En la condición $\mathrm{CCr}$ recibieron monohidrato de creatina (12 gr x d x 7 d), dividido en 2 dosis diarias. De igual dosis y duración se suministró un carbohidrato para la condición PL. Se registró el tiempo en cada sprint de las diferentes condiciones y seguidamente se utilizó una ANOVA de dos vías (condiciones x número de sprint) para mediciones pareadas. El análisis estadístico identificó una interacción significativa $(\mathrm{p}<0.05)$, posteriormente un análisis de seguimiento mostró que las condiciones $\mathrm{CT}$ y $\mathrm{CCr}$ ocasionaban la interacción $(\mathrm{p}<0.05)$. Un segundo análisis de efecto simple indico diferencias significativas $(\mathrm{p}<0.05)$, en todos los sprints. El post hoc de Tukey evidenció que las condiciones $(\mathrm{CCr}, \mathrm{PL})$, eran siempre diferentes a la condición CT, no así entre ellas. Excepto en el sprint 1: CT ( $\overline{\mathrm{x}}=37.03$ $\pm .77 \mathrm{~s})$ y $\operatorname{CCr}(\overline{\mathrm{x}}=35.87 \pm .99 \mathrm{~s})(\mathrm{p}<0.05)$; y entre $\operatorname{CCr}(\overline{\mathrm{x}}=35.87 \pm .99 \mathrm{~s})$ y $\operatorname{PL}(\overline{\mathrm{x}}=36.72 \pm .54 \mathrm{~s})$. Hubo efectos simples de los tratamientos sobre el tiempo promedio $(\mathrm{p}=.001)$ y del número de sprint $(\mathrm{p}=$ .000 ), pero no fueron confirmados por la interacción. En conclusión hubo una mejora clara en la condición $\mathrm{CCr}$ solo en el primer sprint 1 , y una tendencia a retardar la fatiga con la condición PL. PALABRAS CLAVES: Creatina, atletismo, nutrición deportiva, placebo, sprints.
\end{abstract}

\section{INTRODUCCIÓN}

A la Creatina (Cr), que fue descrita por primera vez en 1832 por Chevreul (GuerreroOliveros, \& Wallimann, 1998), se le han atribuido efectos ergogénicos en el ejercicio físico. Por ejemplo, existe evidencia de que la $\mathrm{Cr}$ podría mejorar la velocidad, potencia, fuerza y el trabajo físico, además de estimular cambios en la composición corporal, tanto en hombres como en mujeres (Gotshalk, Volek, Staron, Denegar, Hagerman, \& Kraemer, 2002; Mihic, MacDonald, McKenzie, \& Tarnopolsky, 2000; Rico-Sanz y Mendez,
2000; Mujika, Padilla, Ibáñez, Izquierdo, \& Gorostiaga, 2000; Becque, Lochmann, \& Melrose, 2000).

Esta proteína, formada básicamente por los aminoácidos arginina, lisina y metionina, es sintetizada en diferentes partes del cuerpo, principalmente en el hígado, el páncreas y los riñones (American College of Sports Medicine, 2000). Se ha estimado que las personas podrían utilizar la $\mathrm{Cr}$ a una tasa de 2 gr/día, y que en un hombre sano pueden existir aproximadamente $125 \mathrm{mmol} / \mathrm{kg}$ de $\mathrm{Cr}$, en músculo seco (Juhn, 1999; Greenhaff, 1995). Parece que las necesidades básicas de Cr pueden ser cubiertas por la dieta diaria, 
como por ejemplo cuando se consume carne y pescado. Sin embargo, los investigadores muestran que cuando se ingieren dosis de $\mathrm{Cr}$ entre $\operatorname{los} 2-30 \mathrm{~g} \cdot$ día $^{-1}$ durante periodos cortos de suplementación ( $>10$ días), el organismo es capaz de aumentar los niveles totales de $\mathrm{Cr}(\mathrm{Cr}+\mathrm{PCr})$ de un $10-60 \%$ de sus valores iniciales (Juhn, 1999; American College of Sports Medicine, 2000; Poortmans, \& Francaux, 2000; Greenhaff, 1995).

La mejoría en los niveles totales de $\mathrm{Cr}$ producto de protocolos de suplementación, ha servido como explicación para justificar la optimización del rendimiento en el ejercicio intenso $\left(90-100 \%\right.$ del $\left.\mathrm{VO}_{2 \text { máx }}\right)$, compuesto de muchas repeticiones y de larga duración (Preen, Dawson, Goodman, Lawrence, Beilby, \& Ching, 2001; Engelhardt, Neumann, Berbalk, \& Reuter, 1998). Estos investigadores mencionan que los niveles aumentados de $\mathrm{Cr}$ ayudaron acelerar la capacidad de restaurar los niveles de $\mathrm{PCr}$ durante las pausas o la realización de ejercicio submáximo, convirtiendo más energía (i.e., ATP) en menor tiempo, lo que es considerado como un efecto beneficioso para las siguientes repeticiones anaeróbicas.

Otros investigadores habían descrito previamente la relación entre el aumento en los niveles totales de $\mathrm{Cr}$ y la mejoría en la resíntesis de $\mathrm{PCr}$ durante los descansos cuando se realizaba ejercicio contra resistencia (Greenhaff, Bodin, Soderlund, \& Hultman, 1994; Smith, Montain, Matott, Zientara, Jolesz, \& Fielding, 1998).

El efecto de la suplementación con $\mathrm{Cr}$ se ha estudiado en diferentes disciplinas deportivas: ciclismo estático (Rockwell, Walberg-Rankin, \& Toderico, 2001; Prevost, Nelson, \& Morris, 1997), natación (Burke, Pyne, \& Telford, 1996; Grindstaff, Kreider, Bishop, Wilson, Wood, Alexander, \& Almada, 1997; Peyrebrune, Nevill, Donaldson, \& Cosford, 1998), pruebas de remos (Rossiter, Cannell, \& Jakeman, 1996), ejercicios de Kayak en máquinas ergométricas (McNaughton, Dalton, \& Tarr, 1998), en atletismo estático (Bosco et al.,
1997) y atletismo en pista (Redondo, Dowling, Graham, Almada, \& Williams, 1996; Aaserund, Gramvik, Olsen, \& Jensen, 1998; Terrillion, Kolkhorst, Dolgener, \& Joslyn, 1997). Los resultados son mixtos y contradictorios en todas las disciplinas; es decir, en algunos estudios se apoya el uso de la creatina para mejorar el rendimiento físico; mientras que en otros estudios no.

Durante repeticiones máximas (sprints) que contemplen la carrera de atletismo, solamente Bosco et al. (1997), Aaserud et al. (1998), y Mujika et al. (2000), reportan efectos positivos de la suplementación con $\mathrm{Cr}$ en el tiempo de carrera, es decir, reduciendo los tiempos. En los protocolos de ejercicio de los estudios anteriores, excepto por el de Bosco et al. (1997), realizaron los sprints de forma interválica y repetitiva. La principal explicación de los resultados de Mujika et al. (2000), consistió en que hubo menor participación del sistema glucolítico, debido a una mayor disposición de ATP durante el ejercicio, lo que pudo haber ocurrido por una pronta recuperación de las fuentes energéticas de fosfato.

La evidencia existente hasta el momento indica que puede haber mejorías en el rendimiento físico durante el ejercicio intermitente y prolongado de ciclismo (Preen et al., 2001; Engenlhardt et al., 1998), y en el ejercicio intermitente pero no prolongado del atletismo (Mujika et al., 2000; Aaserud et al., 1998).

Bajo este contexto, hace falta estudiar si el uso de $\mathrm{Cr}$ podría optimizar el rendimiento anaeróbico en el ejercicio intermitente y prolongado en atletismo. En un estudio previo, Gutiérrez, Moncada y Salazar (2002) mencionaron la necesidad de aislar la disciplina del atletismo de un protocolo de ejercicio mixto (natación, ciclismo, atletismo) que se prolongó por más de una hora. Hay evidencia de cómo los protocolos con fatiga previa al ejercicio máximo, encuentran efectos positivos de la suplementación con $\mathrm{Cr}$ (Engenlhardt et al., 1998), y también lo hay con protocolos de ejercicio máximo, intermitente y prolongado (Preen et al., 
2001). Parece importante estudiar más el efecto de la $\mathrm{Cr}$, pero en protocolos que sometan al cansancio a los sujetos $(+1 \mathrm{hr}$ de ejercicio). Por ello, el propósito de este estudio fue examinar el efecto de la $\mathrm{Cr}$ sobre el rendimiento anaeróbico, en un protocolo con fatiga previa a ejercicio intermitente $\mathrm{y}$ máximo de atletismo.

\section{METODOLOGÍA}

\section{Participantes}

En este estudio participaron seis hombres físicamente activos, estudiantes de la carrera en Educación Física, con un peso $\bar{x}=76.25$ $\pm 4.88 \mathrm{~kg}$., talla $\bar{x}=178.96 \pm 5.02 \mathrm{~cm}$., y una edad $\bar{x}=21.4 \pm 1.13$ años.

Inicialmente, los participantes fueron examinados para conocer su estado de salud y avalar su participación en el estudio. Seguidamente, cada participante firmó un formulario de consentimiento informado para participar en el estudio. Posteriormente respondieron un cuestionario, concluyendo que ninguno había utilizado algún tipo de suplemento nutricional o estimulante para mejorar su desempeño físico, antes o al inicio del estudio. También se les indicó que no podían consumir otras sustancias suplementarias durante la realización del estudio.

\section{Instrumentos de medición}

Cada participante portaba un monitor del ritmo cardiaco marca Polar ${ }^{\circledR}$. Los tiempos en los sprints, fueron medidos con 6 cronómetros marca Casio ${ }^{\circledR}$ con capacidad para grabar hasta 100 registros cada uno. Se utilizó una pista atlética de asfalto de $400 \mathrm{~m}$ con 8 carriles. Las salidas en los sprints se dieron con una pistola de aire. El peso corporal fue medido con una balanza electrónica

\section{Procedimientos}

Para una mejor comprensión, los procedimientos se dividieron en tres partes: a) diseño experimental; b) protocolo de suplementación; c) protocolo de ejercicio.
Diseño experimental: En un diseño de grupo único, con ciego simple (los sujetos desconocían la condición asignada en cada sesión), de medidas repetidas y con aleatorización del orden de las condiciones para cada sujeto. Las condiciones experimentales fueron: a) control $(\mathrm{CT})$; b) suplementación con creatina $(\mathrm{CCr})$; y c) suplementación con placebo (PL). Cada condición experimental estuvo separada por 30 días.

Protocolo de suplementación: En este estudio, se utilizó un protocolo de suplementación similar a otros previamente publicados (Preen et al 2001; Engenlhardt et al., 1998; Redondo et al., 1996; Terrillion et al., 1997; Mujika et al 2000).

Así, durante la condición CT los participantes no recibieron ningún tipo de tratamiento (suplemento o PL) durante 30 días. Sin embargo, se les midió el rendimiento anaeróbico bajo las mismas condiciones de ejercicio, estandarizadas para todas las condiciones.

La condición $\mathrm{CCr}$, consistió en dar una sobrecarga durante 7 días previos, de monohidrato de creatina marca Labrada ${ }^{\circledR}$ $\left(12 \mathrm{gr} / \mathrm{d}^{-1}\right)$, dividido en 2 dosis diarias, una en la mañana y otra en la noche. Cada dosis se disolvió en $250 \mathrm{ml}$ de jugo de uvas.

La condición PL consistió en suministrar glucosa en igual dosis a la $\mathrm{CCr}$, disuelta también en jugo de uvas. Estudios clásicos y recientes han utilizado también carbohidratos como PL (Gotshalk et al., 2002; Bosco et al., 1997; Greenhaff et al., 1994).

Protocolo de ejercicio: El requisito para observar el efecto de la $\mathrm{Cr}$ en el rendimiento físico, consistía primero en ocasionar fatiga a los participantes por medio de ejercicio submáximo de carrera pedestre (6 repeticiones X $15 \mathrm{~min}$ ), y después un tiempo determinado $(+1 \mathrm{hr})$, medir en cada participante el rendimiento durante ejercicio anaeróbico y repetitivo $(6$ sprints de atletismo). El procedimiento experimental, que se estandarizó en cada ocasión, es descrito a continuación. Los participantes fueron citados a las 7:30 am en el complejo 
deportivo de la localidad. Dos días antes de la realización de las pruebas no realizaron ningún ejercicio físico; tampoco el día anterior consumieron sustancias con cafeína. La cena fue equiparada en kilocalorías sin variar su alimentación.

Después de la llegada de los participantes al complejo deportivo, éstos realizaron un calentamiento breve, sin incluir ejercicios intensos ni prolongados. Seguidamente procedieron a realizar 6 repeticiones de 15 minutos cada una de ejercicio submáximo $\left(60-85 \% \quad \mathrm{FC}_{\text {máx }}\right)$, con una pausa de $5 \mathrm{~min}$ entre cada una. La ingesta de agua fue ad libitum, antes y durante la prueba.

Finalizado el ejercicio submáximo se procedió a medir el rendimiento anaeróbico en el atletismo, con la ejecución de 6 sprints (200 $\mathrm{m}$ pista) realizados a máxima intensidad $\left(>90 \% \mathrm{FC}_{\text {máx }}\right.$ ). El tiempo de la pausa (3 min 45 s) entre cada sprint, fue establecido con base a un valor de la $\mathrm{FC} \leq 100 \mathrm{lat} \cdot \mathrm{min}^{-1}$, que promediaron los participantes durante una medición piloto.

La variable dependiente fue el tiempo realizado en cada uno de los 6 sprints.

\section{Análisis Estadístico}

El análisis de los datos se realizó con el Paquete Estadístico para las Ciencias Sociales (SPSS, 1998). Los datos se presentan como promedio $(\bar{x})$ y la desviación estándar $( \pm$ $D S)$. Se utilizó el análisis de varianza (ANOVA) de dos vías para medidas pareadas en ambos factores (3 condiciones $\mathrm{x} 6$ sprints). Cuando existió interacción significativa, se realizó el análisis de efectos simples correspondiente, seguido de un post hoc de Tukey. Finalmente se determinó la proporción de varianza explicada por medio del cálculo estadístico de omega al cuadrado $\left(\omega^{2}\right)$.

\section{RESULTADOS}

El ANOVA reveló una interacción significativa $(p<0.05)$ entre las condiciones experimentales y el número de sprints $\left(\omega^{2}=\right.$ $3.76 \%$ ). También reveló un efecto de la condición experimental sobre el tiempo promedio en los sprints $(p=.001)\left(\omega^{2}=\right.$ $37.12 \%)$. Un post hoc de Tukey a los promedios marginales (efectos principales) mostró diferencias $(p<0.05)$ entre la condición CT $(\overline{\mathrm{x}}=37.95 \pm 0.78 \mathrm{~s})$ con la condición $\mathrm{CCr}(\overline{\mathrm{x}}=36.74 \pm .92 \mathrm{~s})$ y con PL $(\overline{\mathrm{x}}=36.66 \pm .80 \mathrm{~s})$.

Otro efecto principal muestra diferencias en los tiempos con respecto al número de sprint $(p=.000)\left(\omega^{2}=13.32 \%\right)($ Tabla 1$)$.

El primer análisis de efectos simples reveló que las condiciones $\mathrm{CT}$ y $\mathrm{CCr}$ eran diferentes entre sí $(p<0.05)$, ocasionando la interacción, no así con la condición PL $(p>$ $0.05)$. El segundo análisis de seguimiento reveló que las diferencias significativas $(p<$ 0.05 ) en el tiempo ocurrieron durante todos los sprints, contrario a los efectos principales.

Los análisis post hoc de Tukey mostraron diferencias estadísticamente significativas $(p$ $<0.05)$ en el sprint 1 entre CT $(\bar{x}=37.03 \pm$ $.77 \mathrm{~s})$ y $\mathrm{CCr}(\overline{\mathrm{x}}=35.87 \pm .99 \mathrm{~s})$; y entre $\mathrm{CCr}$ $(\bar{x}=35.87 \pm .99 \mathrm{~s})$ y PL $(\bar{x}=36.72 \pm .54 \mathrm{~s})$. En el sprint 2 entre CT $(\bar{x}=37.86 \pm .40 \mathrm{~s})$ y $\mathrm{CCr}(\overline{\mathrm{x}}=36.22 \pm 1.10 \mathrm{~s})(p<0.05)$; también entre CT $(\overline{\mathrm{x}}=37.86 \pm .40 \mathrm{~s})$ y $\operatorname{PL}(\overline{\mathrm{x}}=36.69$ $\pm .82 \mathrm{~s})(p<0.05)$. Para el sprint 3 las diferencias estaban entre $\mathrm{CT}(\overline{\mathrm{x}}=37.97 \pm .67$ s) y $\operatorname{CCr}(\overline{\mathrm{x}}=36.79 \pm .93 \mathrm{~s})(p<0.05)$; también CT $(\overline{\mathrm{x}}=37.97 \pm .67 \mathrm{~s})$ y PL $(\overline{\mathrm{x}}=$ $36.20 \pm 1.06 \mathrm{~s})(p<0.05)$. En el sprint 4 había diferencias entre $\mathrm{CT}(\mathrm{M}=37.98 \mathrm{~s} \pm$ $.61 \mathrm{~s})$ y $\mathrm{CCr}(\overline{\mathrm{x}}=36.99 \pm .53 \mathrm{~s})(p<0.05)$; también entre CT $(\overline{\mathrm{x}}=37.98 \pm .61 \mathrm{~s})$ y PL $(\overline{\mathrm{x}}=36.54 \pm .93 \mathrm{~s})(p<0.05)$. En el sprint 5 las diferencias fueron entre CT $(\overline{\mathrm{x}}=38.41 \pm$ $.65 \mathrm{~s})$ y $\operatorname{CCr}(\overline{\mathrm{x}}=37.16 \pm .55 \mathrm{~s})(p<0.05)$; también entre CT $(\overline{\mathrm{x}}=38.41 \pm .65 \mathrm{~s})$ y PL $(\overline{\mathrm{X}}=36.93 \pm .64 \mathrm{~s})(p<0.05)$. Finalmente en el sprint 6 hubo diferencias entre CT $(\overline{\mathrm{x}}=$ $38.48 \pm .72 \mathrm{~s})$ y $\mathrm{CCr}(\overline{\mathrm{x}}=37.41 \pm .46 \mathrm{~s})(p<$ $0.05)$; también entre CT $(\bar{x}=38.48 \pm .72 \mathrm{~s}) \mathrm{y}$ $\operatorname{PL}(\overline{\mathrm{x}}=36.91 \pm .83 \mathrm{~s})(p<0.05)$. 
Tabla 1. Tiempos en los sprints de atletismo $(6 \times 200 \mathrm{~m})$ en condición control (CT), condición con creatina $(\mathrm{CCr})$ y condición placebo $(\mathrm{PL})$. Los valores representan el promedio $(\bar{x})$ y la desviación estándar ( \pm DS).

\begin{tabular}{|c|c|c|c|c|c|c|}
\hline \multirow{2}{*}{$\begin{array}{l}\text { Condición } \\
\text { experimental }\end{array}$} & \multicolumn{6}{|c|}{ Número de Sprint y tiempo (s) } \\
\hline & 1 & 2 & 3 & 4 & 5 & 6 \\
\hline Control & $37.03 \pm .77$ & $37.83 \pm .86$ & $37.97 \pm .67$ & $37.98 \pm .61$ & $38.41 \pm .65$ & $38.48 \pm .72$ \\
\hline Creatina & $35.87 \pm .99^{\mathrm{ab}}$ & $36.22 \pm 1.10^{\mathrm{a}}$ & $36.79 \pm .93^{\mathrm{a}}$ & $36.99 \pm .53^{\mathrm{a}}$ & $37.16 \pm .55^{\mathrm{a}}$ & $37.41 \pm .46^{\mathrm{a}}$ \\
\hline Placebo & $36.72 \pm .54$ & $36.69 \pm .82^{\mathrm{a}}$ & $36.20 \pm 1.06^{\mathrm{a}}$ & $36.54 \pm .93^{\mathrm{a}}$ & $36.93 \pm .64^{\mathrm{a}}$ & $36.91 \pm .83^{\mathrm{a}}$ \\
\hline$\overline{\mathrm{x}}$ Total & $36.54 \pm .90$ & $36.92 \pm 1.02$ & $36.99 \pm 1.13$ & $37.17 \pm .91$ & $37.50 \pm .88 *$ & $37.60 \pm .93 *$ \\
\hline
\end{tabular}

Figura 1. Cambio en el tiempo $(\Delta \%)$ para la condición control $(\mathrm{CT})$, con creatina (CCr) y placebo $(\mathrm{PL})$ en los 6 sprints de atletismo $(200 \mathrm{~m})$.
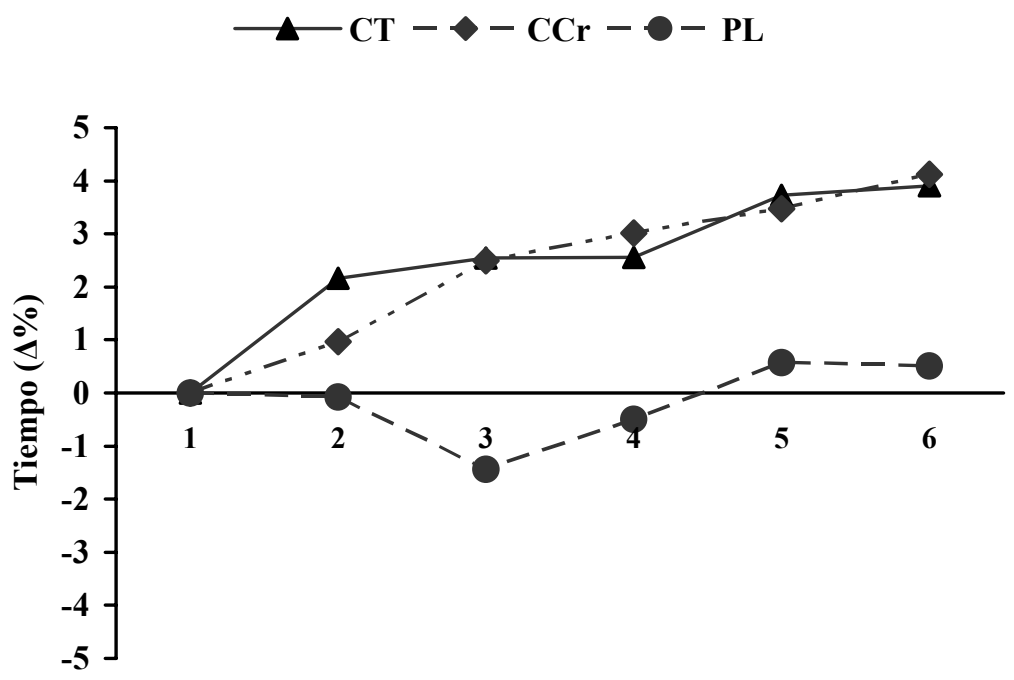

Número de Sprint

\section{DISCUSIÓN}

El propósito de este estudio era investigar si la suplementación con $\mathrm{Cr}$ mejoraba el rendimiento anaeróbico durante esfuerzos máximos en la carrera repetitiva de $200 \mathrm{~m}$ del atletismo, después de ocasionar fatiga con ejercicio prolongado en forma intermitente $(>1 \mathrm{~h})$.

En un estudio previo, Gutiérrez et al. (2002), mostraron que la suplementación con Cr tuvo un efecto positivo básicamente en el atletismo al realizar los últimos intervalos máximos. También se observó un efecto 
placebo, dado que en esa condición el tiempo no fue afectado por el cansancio, contrario a la condición control donde el tiempo se incrementó conforme transcurrían los intervalos.

En el estudio de Gutiérrez et al. (2002), se llega a la conclusión de que era necesario estudiar los tratamientos $\left(\begin{array}{lll}\mathrm{Cr} & \mathrm{y} & \mathrm{PL}\end{array}\right)$ exclusivamente en la disciplina del atletismo, y durante condiciones similares de ejercicio acumulado (+ $1 \mathrm{hr})$.

Al finalizar este estudio, se identificó de igual forma que en la investigación de Gutiérrez et al. (2002) un efecto mixto, tanto de la suplementación con CCr como PL. Sin embargo, existió una diferencia importante: la mejora en el rendimiento durante el tratamiento experimental $(\mathrm{CCr})$ ocurrió de forma clara, solamente en el sprint 1 (Tabla 1), contrario a lo observado en el estudio anterior.

Por otra parte, el desempeño físico durante la condición placebo fue muy similar a la observada en el estudio de Gutiérrez et al. (2002). En la figura 1, puede observarse que el rendimiento en términos de porcentaje de cambio es muy estable, a excepción del sprint 3 donde hubo una ligera mejoría. No obstante, al comparar la condición PL con las otras condiciones se nota un claro contraste. El efecto placebo en los procesos psicológicos amerita mayor investigación, debido a que los datos de este estudio indican que en la condición placebo los sujetos no evidenciaron ningún síntoma de cansancio y pudieron mantener su rendimiento estable durante los seis sprints, aspecto que no ocurrió con el rendimiento en las otras dos condiciones.

Parece importante para futuros estudios valorar también la magnitud que tiene el tratamiento con PL sobre el rendimiento. En los estudios de Redondo et al. (1996) y Terrillion et al. (1997), no se encontraron interacciones significativas al analizar el efecto de la $\mathrm{Cr}$ sobre el atletismo, pero sí una tendencia a que las diferencias en el post-test entre el grupo PL y CCr fueron mínimas. Esto sugiere que aunque no hubo un cambio significativo en estos estudios (Redondo et al., 1996; Terrillion et al., 1997), de pre a post-tratamiento con Cr, sí cambió de forma parecida el rendimiento posterior a la suplementación con Cr o PL.

Mujika et al. (2000) encontraron efectos positivos del uso de $\mathrm{Cr}$, durante la primera repetición de varios sprints de carrera en distancia corta $(5 \mathrm{~m})$. Pero también se observa gráficamente que los tiempos en el pos-test para ambas condiciones (CCr y PL) en el primer sprint $(5 \mathrm{~m})$ son prácticamente iguales, y también semejantes en el promedio general posterior a finalizar los 6 sprints. Esta situación no fue contemplada en la discusión del artículo.

En otro estudio (Aaserud et al., 1998), hay una condición similar a la del estudio de Mujika et al. (2000). Aunque en ambos no se reportaron las tablas con los promedios y desviaciones estándar, sí es posible observar en los gráficos que el cambio en el rendimiento pos-tratamiento para los grupos PL y $\mathrm{CCr}$ fue semejante. Tampoco se comenta este hecho en la discusión.

El análisis de la interacción en este estudio reveló que en todos los sprints había diferencias en el tiempo, contrario a lo observado en los efectos principales. También identificó que las diferencias ocurrieron con respecto a la condición CT excepto en el sprint 1 (Tabla 1). Esto es distinto a lo mostrado en los efectos principales, que mostraron que en la condición $\mathrm{CCr}$ y en la condición PL eran siempre iguales en su tiempo promedio.

Por otra parte, al observar el gráfico 1, se interpreta que las condiciones $\mathrm{CCr}$ y $\mathrm{CT}$ tuvieron una magnitud de cambio similar. Pueden plantearse dos explicaciones a los resultados encontrados. Por una parte, se debería considerar un efecto de retención de condición con $\mathrm{Cr}$, ya que aunque existió aleatorización pudo haber permanencia del tratamiento de Cr. Otra explicación estaría en considerar que la $\mathrm{Cr}$ sí mejora aspectos fisiológicos (Greenhaff et al., 1994; Smith et al., 1998; Kreis et al., 1999) que intervienen en el rendimiento (mayor resíntesis de ATP), 
pero también los procesos psicológicos producidos por PL pueden tener una magnitud parecida a la $\mathrm{Cr}$.

Existe cierta evidencia que indica que el uso de la Cr optimiza el rendimiento físico durante protocolos con ejercicio mixto (aeróbico - anaeróbico) que se extendió por más de una hora (Preen et al., 2001; Engenlhardt et al., 1998). Sin embargo, esta evidencia está concentrada en el ejercicio sobre cicloergómetros (ciclismo estacionario). En el caso del atletismo los estudios están limitados al ejercicio intermitente y de corta duración, además las conclusiones o hallazgos son controversiales. Es decir, en algunos estudios se han reportado mejorías en el rendimiento (Mujika et al., 2000; Aaserud et al., 1998; Bosco et al., 1997) mientras que en otros estudios no (Redondo et al., 1996; Terrillion et al., 1997).

Se ha mencionado la similitud que hay entre los tratamientos (Cr y PL) y también la inconcistencia en los resultados de los estudios. La respuesta podría encontrarse en otras variables extrañas que no han sido analizadas o clasificadas. En conclusión, parece necesario aplicar la técnica metaanalítica y así estimar la verdadera relevancia del uso de la $\mathrm{Cr}$ como suplemento ergógeno. Según Salazar, Petruzzello, Landers, Etnier, y Kubitz (1992), a través del meta-análisis se puede resumir la información y cuantificar la magnitud que tiene los tratamientos sobre el rendimiento físico.

Sin embargo en este caso es necesario clasificar las magnitudes de las condiciones PL y $\mathrm{Cr}$ de acuerdo con su diseño estadístico (pareados o independientes) dado que podrían existir evidencias importantes que ayuden a clarificar el conflicto.

\section{REFERENCIAS}

Aaserund, R., Gramvik, P., Olsen, S. R., \& Jensen, J. (1998). Creatine supplementation delays onset of fatigue during repeated bouts of sprint running. Scandinavian Journal of Medicine and Science in Sportse, 8, 247-251.

American College of Sports Medicine Roundtable. (2001). The physiological and health effect of oral creatine supplementation. Medicine and Science in Sports and Exercise, 32(3), 706717.

Becque, M. D., Lochmann, J. D., \& Melrose, D. R. (2000). Effects of oral creatine supplementation on muscular strength and body composition. Medicine and Science in Sports and Exercise, 32(3), 654-658.

Bosco, C., Tihanyi, J., Pucspk, J., Kovacs, I., Gabossy, A., Colli, R., Pulvirenti, G., Tranquilli, C., Foti, C., Viru, M., \& Viru, A. (1997). Effect of oral creatine supplementation on jumping and running performance. International Journal of Sports Medicine, 18(5), 369-372.

Burke, L. M., Pyne, D. B., \& Telford, R. D. (1996). Effect of oral creatine supplementation on single-effort sprint performance in elite swimmers. International Journal of Sport Nutrition, 6, 222-233.

Engelhardt, M., Neumann, G., Berbalk, A., \& Reuter, I. (1998). Creatine supplementation in endurance sports. Medicine and Science in Sports and Exercise, 30(7), 1123-1129.

Gotshalk, A. L., Volek, S. J., Staron, S. R., Denegar, R. C., Hagerman, C. F. \& Kraemer, J. W. (2002). Creatine supplementation improves muscular performance in older men. Medicine and Science in Sports and Exercise, 34(3), 537543.

Guerrero-Oliveros, M. L., \& Wallimann, T. (1998). Creatine supplementation in health and disease. Effects of chronic creatine ingestion in vivo: Down-regulation of the expression of creatine transporter isoforms in skeletal muscle. Molecular Cell Biochemistry, 184, 427-437.

Gutiérrez, O., Moncada, J., \& Salazar, W. (2002). Efecto del consumo de creatina sobre la ejecución de destrezas realizadas en forma intermitente y de duración prolongada. Revista del Ciencias del Ejercicio y la Salud, 2(1), 23-30.

Greenhaff, P. L. (1995). Creatine and application as an ergogenic aid. International Journal of Sport Nutrition, 5, S100-S110.

Greenhaff, P. L., Bodin, K., Soderlund, K., \& Hultman, E. (1994). Effect of oral creatine supplementation on skeletal phosphocreatine resynthesis. American Journal of Phsiology, 266(29), E725-E730.

Grindstaff, P. D., Kreider, R., Bishop, R., Wilson, M., Wood, L., Alexander, C., \& Almada, A. (1997). Effects of creatine supplementation on repetitive sprint performance and body composition in competitive swimmers. International Journal of Sport Nutrition, 7, 330-346.

Juhn, M. S. (1999). Oral creatine supplementation: separating fact from hype. The Physician and SportsMedicine, 27(5), 47-61. 
Kreis, R., Kamber, M., Koster, M., Felblinger, J., Slotboom, J., Hoppeler, H., \& Boesch, C. (1999). Creatine supplementation - Part II: in vivo magnetic resonance spectroscopy. Medicine and Science in Sports and Exercise, 31(12), 1770-1777.

Mihic, S., MacDonald, J. R., McKenzie, S., \& Tarnopolsky, M. A. (2000). Acute creatine loading increases fat-free masss, but does not affect blood pressure, plasma creatinine, or $\mathrm{CK}$ activity in men and women. Medicine and Science in Sports and Exercise, 32(2), 291296.

Mujika, I., Padilla, S., Ibáñez, J., Izquierdo, M., \& Gorostiaga, E. (2000). Creatine supplementation and sprint performance in soccer players. Medicine and Science in Sports and Exercise, 32(2), 518-525.

McNaughton, L. R., Dalton, B., \& Tarr, J. (1998). The effects of creatine supplementation on hingintensity exercise performance in elite performer. European Journal Appling Physiology, 78(3), 236-240.

Peyrebrune, M. C., Nevill, M. E., Donaldson, F. J., \& Cosford, D. J. (1998). The effects of oral creatine supplementation on performance in single and repeated sprint swimming. Journal of Sport Sciences, 16, 271-279.

Poortmans, R. J., \& Francaux, M. (2000). Adverse effects of creatine supplementation. Fact or fiction. Sports Medicine, 30(3), 155-170.

Preen, D., Dawson, B., Goodman, C., Lawrence, S., Beilby, J., \& Ching, S. (2001). Effect of creatine loading on long-term sprint exercise performance and metabolism. Medicine and Science in Sports and Exercise, 33(5), 814821.

Prevost, M. C., Nelson, A. G., \& Morris, G. S. (1997). Creatine supplementation enhances intermittent work performance. Research Quarterly for Exercise and Sports, 68(3), 233240

Redondo, D. R., Dowling, E. A., Graham, B. L., Almada, A. L., \& Williams, M. H. (1996). The effect of oral creatine monohydrate supplementation on running velocity. International Journal of Sport Nutrition, 6, 213-221.

Rico-Sanz, J., y Mendez, M. M. (2000). Creatine enhances oxygen uptake and performance during alternating intensity exercise. Medicine and Science in Sports and Exercise, 32(2), 379-385.

Rockwell, J. A., Walberg-Rankin, J., \& Toderico, B. (2001). Creatine supplementation affects muscle creatine energy restriction. Medicine and Science in Sports and Exercise, 33(1), 6168.

Rossiter, H. B., Cannell, E. R., \& Jakeman, P. M. (1996). The effect of oral creatine supplementation on the 1000 -m performance of competitive rowers. Journal of Sports Sciences, 14, 175-179.

Salazar, W., Petruzzello, S. J., Landers, D. M., Etnier, J. L., \& Kubitz, K. A. (1992). Meta-analytic techniques in exercise psychology. En Seraganian, P. (Ed). Advances in Exercise Psychology. John Wiley \& Sons.

Smith, S. A., Montain, S. J., Matott, R. P., Zientara, G. P., Jolesz, F. A., \& Fielding, R. A. (1998). Creatine supplementation and age influence muscle metabolism during exercise. Journal of Applied Physiology, 85(4), 1349-1356.

Statistical Package for Social Sciences. (1998). SPSS advanced statistics 8.0. Chicago, IL: SPSS.

Terrillion, K. A., Kolkhorst, F. W., Dolgener, F. A., \& Joslyn, S. J. (1997). The effect of creatine supplementation on two 700-m máximo running bouts. International Journal of Sport Nutrition, 7, 138-143. 